\title{
Infants with Intrauterine Growth Restriction Have Impaired Formation of Docosahexaenoic Acid in Early Neonatal Life: A Stable Isotope Study
}

\author{
ADOLFO LLANOS, YUHONG LI, PATRICIA MENA, \\ NORMAN SALEM, JR., AND RICARDO UAUY \\ Institute of Nutrition and Food Technology [A.L., P.M., R.U.], Santiago, Casilla 138-11, Chile; \\ Laboratory of Membrane Biochemistry and Biophysics [Y.L., N.S.], National Institute on Alcohol Abuse \\ and Alcoholism, National Institutes of Health, Rockville, MD 20852; Neonatology Unit Hospital Sotero \\ del Rio [P.M.], Santiago, 820-7257, Chile; and London School of Hygiene and Tropical Medicine [R.U.], \\ London WC 1E 7HT, United Kingdom
}

\begin{abstract}
ABS
This study evaluated the arachidonic acid (AA) and doco-
sahexaenoic acid (DHA) formation from d5-labeled linoleic acid (d5-LA) and $\alpha$-linolenic acid (d5-LNA) precursors in infants with intrauterine growth restriction (IUGR) compared with control groups matched by gestational age (GA) or birth weight. We compared DHA and AA formation from deuterated precursors d5-LA and d5-LNA in 11 infants with IUGR with 13 and 25 control subjects who were appropriate for GA and matched by GA and by birth weight, respectively. After an enteral administration of d5-LA and d5-LNA, we determined unlabeled and d5-labeled fatty acids at 24, 48, and $96 \mathrm{~h}$ in plasma. Absolute concentrations and area under the curve (AUC) over the 96-h study were used for analysis. Absolute concentration of d5-DHA and the product/precursor ratio of the d5-labeled AUCs indicated a less active DHA formation from LNA in infants with IUGR compared with their GAmatched (2-fold) and birth weight-matched (3-fold) control subjects. The ratios of eicosapentaenoic and n-3 docosapentaenoic acid to DHA were also affected. Similar evaluation for the $n-6$ series was not significant. DHA metabolism is affected in infants with IUGR; the restricted DPA to DHA conversion step seems to be principally responsible for this finding. (Pediatr Res 58: 735-740, 2005)
\end{abstract}

Abbreviations

AA, arachidonic acid

AGA, appropriate for gestational age

AUC, area under the curve

DHA, docosahexaenoic acid

d5-LA, deuterated linoleic acid

d5-LNA, deuterated $\alpha$-linolenic acid

DPA, docosapentaenoic acid

EFA, essential fatty acids

EPA, eicosapentaenoic acid

GA, gestational age

IUGR, intrauterine growth restriction

LA, linoleic acid

LCP, long-chain polyunsaturated fatty acids

LNA, $\alpha$-linolenic acid

PGC-1, peroxisome proliferator-activated receptor- $\gamma$ coactivator-1

PPAR, peroxisome proliferator-activated receptor

SGA, small for gestational age
Intrauterine growth restriction (IUGR) is the result of the failure of the placenta to provide the necessary nutrients required by the fetus to maintain adequate growth (1). During the last trimester of gestation, there is a significant accumulation of long-chain polyunsaturated fatty acids (LCPs) in the fetus and

Received August 25, 2004; accepted January 26, 2005.

Correspondence: Adolfo Llanos, M.D., INTA U de Chile Macul 5540 Santiago, Chile; e-mail: allanos@inta.cl.

Supported by Fondecyt grant 1990078.

DOI: 10.1203/01.PDR.0000180542.68526.A2 an increase in the relative content of both n- 6 and n-3 LCPs in the human brain and retina. Fetal tissue content of LCPs is dependent on maternal intake and on an adequate placental transfer. Thus, a greater risk for restricted in utero LCPs supply may occur in pregnancies that are complicated with abnormal placental function affecting nutrient transfer. Data derived from IUGR animal models indicate that placental insufficiency is associated with abnormalities in fetal lipid metabolism of skeletal muscle and liver (2,3). Cetin et al. (4) reported that fetuses with IUGR have a lower proportion of long-chain n-6 and n-3 fatty acids, arachidonic acid (AA), and docosahexae- 
noic acid (DHA) relative to the precursors linoleic acid (LA) and $\alpha$-linolenic acid (LNA) in fetal blood in comparison with maternal blood than is found in control infants who are appropriate for gestational age (AGA). Studies that have evaluated essential fatty acids (EFA) status in infants with IUGR, most of them premature, compared with control subjects using umbilical cord blood, the umbilical artery-vein wall, or the placenta reported lower EFA status in infants with IUGR (5-7). Studies in term infants that included infants who were small for gestational age (SGA) failed to corroborate the findings described in preterm infants with IUGR, reporting an even higher biochemical EFA status in infants who were classified as SGA compared with their large for GA counterpart (8). This served to highlight the vulnerability of the maternal/placental supply of EFA to the fetus not only as a result of poor maternal intake and/or placental transfer as a result of placental insufficiency but also of the increased demands as a result of higher EFA accretion by the heavier fetus.

Several studies in human neonates have used stable isotopes, ${ }^{2} \mathrm{H}_{5^{-}}$or ${ }^{13} \mathrm{C}$-labeled LA or LNA, to assess in vivo metabolic conversion reflecting EFA elongation and desaturation. These studies have provided clear evidence that term and preterm infants are able to synthesize AA and DHA from their respective dietary EFAs, LA and LNA, respectively, albeit in small amounts $(9,10)$. Formation of LCPs in infants with IUGR has not been characterized. The objective of this study was to evaluate the AA and DHA formation from d5-labeled LA and LNA precursors in infants with IUGR compared with control groups that were matched by GA or birth weight.

\section{METHODS}

Between March 1994 and October 2001, preterm and full-term infants who were admitted to the neonatal unit at Hospital Sótero del Río in Santiago were invited to participate in the study to evaluate the effect of birth weight and GA on LCP formation in neonates, excluding those with major malformations or those who were unable to receive the enteral dose of tracer fatty acids for any reason (metabolic disorders or maternal diabetes). GA was assessed by last menstrual period or using age of conception on the basis of early ultrasound and confirmed by the modified Ballard evaluation conducted soon after birth (11). They were appropriate for GA (AGA) according to the Lubchenco standard or considered to have IUGR on the basis of fetal ultrasound with postnatal confirmation of SGA defined as weight below the 10th percentile for GA and clinical evidence $(12,13)$. Most preterm infants studied presented with mild to moderate forms of respiratory distress; term infants often had mild forms of hypoxic insult or transient respiratory problems. Infants who were selected for these analyses had the isotope administered in the first few days of life and not beyond the first 2 wk of life. Feeding was generally started within the first $2 \mathrm{~d}$ after birth, and the type of feeding varied depending on the mothers' willingness to provide breast milk. When breast milk was not available, term infants received Nan (Nestle), a formula that is devoid of LCP and contains LA $11.7 \%$ and LNA $1.1 \%$ of total lipids. Preterm infants received either PreNan (Nestle) or Similac Special Care (Ross), both of which were devoid of LCP and had EFA content of LA $12.8 \%$ and LNA $1.1 \%$, and LA $12.6 \%$ and LNA $1.2 \%$ of total lipids, respectively (14). DHA/AA content in breast milk from Chilean mothers after $15 \mathrm{~d}$ of lactation range from 0.4 to $0.7 \%$ DHA, whereas AA is $0.5-0.8 \%$ of total fatty acid weight; the ratio of AA to DHA is close to 1 . Patients who could not be fed enterally received parenteral glucose starting soon after birth; crystalline amino acids were added by day 3 of life. None of the patients included in this study received i.v. lipids, before or during the study period. Written informed consent was obtained by a trained research nurse supervised by a neonatologist shortly after birth or soon after feedings were commenced. The study was approved by INTA's ethics committee and by the research committee at Hospital Sótero del Río that is responsible for supervising research involving human subjects. The protocol was also approved by the National Institutes of Health/National Institute on Alcohol Abuse and Alcoholism Institutional Review Board and received a
Single Project Assurance from the Office for the Protection from Research Risks, Department of Health and Human Services, for international collaboration.

Isotope administration. Patients received $50 \mathrm{mg} / \mathrm{kg}$ each of deuterated linoleic acid (d5-LA) and deuterated $\alpha$-linolenic acid (d5-LNA) as ethyl esters provided by Cambridge Isotope Labs (Andover, MA) by the nasogastric route ranging from the first to the 15 th day of life. Blood was drawn $(0.5 \mathrm{~mL})$ from a peripheral vein or umbilical catheter into a tube that contained EDTA as anticoagulant at baseline and at 24, 48, and $96 \mathrm{~h}$ after dosing (baseline sample was discontinued after the first five patients presented nondetectable concentrations of interfering substances for the d5-labeled fatty acids measured). Plasma was separated by centrifugation shortly after sampling, aliquotted, and kept frozen until lipids were analyzed.

Fatty acid and deuterated fatty acid metabolite assay. The method for fatty acid analysis has been published but is described briefly (15); lipids were extracted from $100 \mu \mathrm{L}$ of plasma using the method of Folch et al. (16) adding $50 \mu \mathrm{g}$ of BHT per sample to prevent oxidation. Fatty acids were methylated using boron trifluoride-methanol according to Morrison and Smith (17) as modified by Salem et al. (18). Quantification was achieved by capillary gas chromatography (model 5890; Hewlett Packard, Wilmington, DE) using flame ionization detection. Samples were air-shipped on dry ice to the National Institute on Alcohol Abuse and Alcoholism Laboratory of Membrane Biochemistry and Biophysics for measurements of the concentrations of d5-fatty acids as previously described (15). A model 5973 Mass Spectrometer with Model 6890 Gas Chromatograph (Agilent, Wilmington, DE) using a FFAP bonded-phase capillary column $(60 \mathrm{~m} \times 0.25 \mathrm{~mm}$ I.D., film thickness 0.25 $\mathrm{mm}$; J \& W Scientific, Folsom, CA) served to separate the d5-labeled fatty acids from the unlabeled products of LA and LNA elongation/desaturation. Results are expressed in terms of d5-labeled fatty acids concentrations in $\mu \mathrm{g} / \mathrm{mL}$ of plasma at 24,48 , and $96 \mathrm{~h}$. All data were coded and managed using computerized spreadsheets and analyzed in a blinded manner.

The change, appearance or disappearance, over time for the d5-labeled products of interest was measured at given times and also integrated over the $96 \mathrm{~h}$ of the study. The area under the curve (AUC) was determined for each labeled fatty acid by application of a time-integrated algorithm (19) to the plasma concentration-time course curve. For each time point, trapezoidal areas are calculated by a formula in which the area equals one half the sum of the concentrations multiplied by the time interval in hours. The sum of the trapezoids from 0 to $96 \mathrm{~h}$ is the AUC).

Group selection. A total of 80 patients were studied; two previous publications have reported data from 51 patients who were evaluated from 1994 until April $1998(10,15)$. The present analysis was conducted in 30 patients from this group (nine in the IUGR and 21 in the AGA matched control groups) and in an additional 14 patients from 29 studied up to August 2001. Thus, the group being reported now corresponds to the 44 patients who received the isotope before $15 \mathrm{~d}$ of life from the total of 80 patients studied.

The present analysis differs from the previously published work in that it limited inclusion to patients who were studied before $15 \mathrm{~d}$ to minimize the confounding effect of feeding and established two control groups for the IUGR group; one matched by GA and a second group matched by birth weight. Matching criteria were defined as $\pm 100 \mathrm{~g}$ for the birth weight group $(n=25)$ and equal GA expressed in weeks for the GA control group $(n=13)$. The present analysis of the data were based on actual concentration of deuterated precursors and products rather than isotope enrichment to avoid the effect of changes in the unlabeled fatty acids in defining the responses. Finally, we assessed formation of DHA and AA on the basis of product:precursor ratios of AUC over the complete study period rather than as ratios from single time points.

Statistical analysis. Statistical analysis of data included measures of central tendency and distribution. Means and SDs for the relevant variables for the three groups were computed. Specific data points that were identified as outliers from the group data were examined for potential errors in data management or chemical analysis. Comparison among the three groups was performed using a nonparametric test given that the distribution of all evaluated parameters of fatty acid metabolism was nonnormally distributed.

\section{RESULTS}

The birth characteristics and anthropometrics for IUGR and control groups are shown in Table 1. As expected, the IUGR group differs in birth weight by nearly $1000 \mathrm{~g}$ with the GAmatched control and is 4 wk older in gestation relative to the birth weight-matched control group (Table 1). The lack of differences in length, head circumference, and ponderal index 
Table 1. Biodemographic and anthropometric indices of infants included in the three groups

\begin{tabular}{lccc}
\hline & \multicolumn{3}{c}{ Control Groups } \\
\cline { 2 - 4 } & IUGR $(n=11)$ & GA $(n=13)$ & BW $(n=25)$ \\
\hline Gestational Age (wks) & $34.0 \pm 3.4^{*}$ & $34.4 \pm 3.5$ & $30.6 \pm 1.7 \S$ \\
Birth Weight (g) & $1650 \pm 398$ & $2526 \pm 839 \S$ & $1540 \pm 327$ \\
Length (cm) & $41.6 \pm 3.6$ & $46 \pm 3.6 \S$ & $40.7 \pm 2.7$ \\
Head circumference (cm) & $29.8 \pm 2.1$ & $32.8 \pm 2.6$ & $29.5 \pm 2.4$ \\
Ponderal Index (kg/m3) & $22.6 \pm 1.8$ & $25.2 \pm 3.5 \S$ & $22.5 \pm 1.8$ \\
Gender (M/F) & $5 / 6$ & $6 / 7$ & $16 / 9$ \\
\hline
\end{tabular}

* Mean \pm SD. Comparison between IUGR and the GA and BW matched control groups was conducted using One way anova followed by post-hoc test with Bonferroni correction for gestational age, birth weight, length, head circumference and ponderal index. Difference in gender was assessed using pearson Chi-2.

$\S p<0.01$.

between the IUGR study group and the birth weight-matched control group despite the 4-wk difference in gestation indicates that these are infants with symmetric growth restriction as opposed to typical asymmetry observed in term infants with IUGR. This pattern suggests that growth restriction had been sustained over a long period.

The age at study entrance for the GA-matched control group was relatively similar to the IUGR group, whereas the infants in the birth weight-matched control group were older than those in the IUGR group [median (range): IUGR $1 \mathrm{~d}(0-15 \mathrm{~d})$; GAmatched control group $0 \mathrm{~d}(0-10 \mathrm{~d})$; birth weight-matched control group $10 \mathrm{~d}(0-15 \mathrm{~d})]$. Most of the infants included in this analysis were premature (nine of 11 in the SGA group and 33 of 38 in the AGA group). Regarding the feeding received by the patients at the time of evaluation, the majority were not receiving enteral feeds: 10 in the IUGR group, eight in the GA control group, and 10 in the birth weight control group. The type of feeding received by the GA and birth weight control groups was predominantly a mix of breast milk and LCP-free milk formula, with a few of them receiving exclusively formula or breast milk.

Comparison of concentrations of unlabeled (d0) and d5labeled (d5) fatty acids for the $\mathrm{n}-3$ and $\mathrm{n}-6$ series measured at 24,48 , and $96 \mathrm{~h}$ after tracer administration is presented in Table 2. Comparison of infants with IUGR in relation to the two control groups was done using nonparametric tests. The concentration of unlabeled LNA measured at $24 \mathrm{~h}$ is significantly lower in the infants with IUGR when compared with the birth weight control infants, suggesting poor transplacental transfer or lower dietary intake. Similar effects are found in LA concentrations at $24 \mathrm{~h}$, but differences are not significant. This difference disappears by $96 \mathrm{~h}$, indicating possible mobilization from tissue pools or increasing dietary intake. The trend in the infants with IUGR indicates a time-related increase in concentration of the unlabeled n-3 fatty acids, whereas the concentration of LNA metabolite EPA in the two control groups remains stable or slightly decreases. The concentrations of d5-labeled fatty acids of infants with IUGR were not significantly different from those of control groups at 24,48 , and $96 \mathrm{~h}$ for the precursors d5-LNA and d5-LA. The d5-labeled concentration for the main product of interest, DHA in the IUGR group, presents lower values than for their matched control infants. These differences are significant at $24 \mathrm{~h}$ compared with the GA control infants and at 24 and $48 \mathrm{~h}$ for the birth weight counterpart. There was also a significantly greater concentration of plasma d5-AA at $24 \mathrm{~h}$ in the birth weight-matched group compared with the IUGR group.

Table 3 provides the results of $\mathrm{d} 5$-fatty acid integrated over time for all of the fatty acids analyzed. Integration of the plasma concentration-time course curve provides the AUC. The values for the AUC for the d5-labeled $n-3$ and $n-6$ precursors and intermediary metabolite between the IUGR and

Table 2. Unlabeled (d0) and labeled (d5) fatty acid concentration ( $\mu \mathrm{g} / \mathrm{ml}$ plasma) at $24 \mathrm{~h}, 48 \mathrm{~h}$ and $96 \mathrm{~h}$ after isotope fr do

\begin{tabular}{|c|c|c|c|c|c|c|c|}
\hline & \multirow[b]{2}{*}{ Hrs } & \multicolumn{2}{|c|}{ IUGR/SGA $(n=11)$} & \multicolumn{2}{|c|}{ GA Controls $(n=13)$} & \multicolumn{2}{|c|}{ BW Controls $(n=25)$} \\
\hline & & $\mathrm{d} 0$ & $\mathrm{~d} 5$ & $\mathrm{~d} 0$ & $\mathrm{~d} 5$ & d0 & d5 \\
\hline \multirow[t]{2}{*}{$18: 3 n-3$} & 24 & $0.52 \S(0.28-3.01)$ & $0.32(0.10-0.79)$ & $1.04(0.7-4.95)$ & $0.52(0.26-0.75)$ & $4.11 *(0.8-10.29)$ & $0.304(0.14-0.46)$ \\
\hline & 96 & $4.5(2.9-10.93)$ & $0.09(0.02-0.16)$ & $3.32(1.92-8.63)$ & $0.05(0.02-0.09)$ & $4.7(2.11-8.86)$ & $0.02 *(0.01-0.05)$ \\
\hline \multirow[t]{2}{*}{$20: 5 n-3$} & 24 & $2.4(1.47-9.94)$ & $0.09(0.01-0.14)$ & $3.5(2.38-5.7)$ & $0.11(0.06-0.32)$ & $4.84(3.5-9.82)$ & $0.06(0.03-0.21)$ \\
\hline & 48 & $3.47(2.45-7.29)$ & $0.1(0.04-0.1798)$ & $3.17(2.83-7.04)$ & $0.12(0.04-0.18)$ & $5.03(3.25-8.95)$ & $0.04(0.02-0.14)$ \\
\hline & 48 & $28.93(22-37.56)$ & $0.02(0.01-0.0319)$ & $28(16.13-40.33)$ & $0.03(0.02-0.07)$ & $28.14(15.61-39.87)$ & $0.07 *(0.02-0.22)$ \\
\hline & 96 & $27.1(22.68-35.09)$ & $0.05(0.01-0.07)$ & $25.20(17.65-29.47)$ & $0.03(0.02-0.12)$ & $27.2(17.87-35.5)$ & $0.08(0.02-0.12)$ \\
\hline \multirow[t]{3}{*}{$18: 2 n-6$} & 24 & $78.91(38.7-180.7)$ & $0.513(0.19-1.11)$ & $80.19(65.78-258.81)$ & $0.793(0.46-0.99)$ & $258(59-437)$ & $0.61(0.11-0.93)$ \\
\hline & 48 & $117.9(80.19-183.97)$ & $0.46(0.18-0.9995)$ & $144.77(73.4-224.13)$ & $0.52(0.3-1.51)$ & $254.24(72.03-367.52)$ & $0.32(0.05-0.54)$ \\
\hline & 96 & $194.38(128.9-337.58)$ & $0.25(0.08-0.61)$ & $224.95(124-361.32)$ & $0.167(0.07-0.58)$ & $308(118.1-401.29)$ & $0.14(0.05-0.25)$ \\
\hline $20: 3 n-6$ & 24 & $20.05(12.12-35.3)$ & $0.01(0.002-0.01)$ & $23.79(19.05-28.4)$ & $0.017(0.01-0.04)$ & $25.23(19.83-36.42)$ & $0.015(0.01-0.0317)$ \\
\hline
\end{tabular}

$\S$ Values presented as median (p25-p75).

Differences between the unlabeled and d5-labeled fatty acids concentrations at 24 and 96 hours in the Gestational Age matched controls and Birth Weight matched controls compared to the IUGR group are significantly different using a non-parametric test (Kruskal-Wallis).

$* p \leq 0.05$. 
Table 3. Area under the plasma concentration-time course curves for IUGR/SGA and reference groups

\begin{tabular}{|c|c|c|c|c|c|}
\hline & IUGR & GA Controls & $\begin{array}{c}\text { GA } v s \text { IUGR } \\
(p \text { values })^{*}\end{array}$ & BW Controls & $\begin{array}{c}\mathrm{BW} v s \text { IUGR } \\
(p \text { values })\end{array}$ \\
\hline \multicolumn{6}{|l|}{$n-3$} \\
\hline d5-18:3 & $18.12 \S(5.21-37.91)$ & $17.98(8.96-39.89)$ & 0.41 & $12.19(6.40-17.98)$ & 0.31 \\
\hline d5-22:5 & $1.88(0.65-2.65)$ & $1.60(1.04-2.58)$ & 0.77 & $1.70(0.93-2.60)$ & 0.64 \\
\hline d5-22:6 & $2.12(0.61-3.45)$ & $2.94(1.47-7.17)$ & 0.37 & $6.30 *(2.65-17.55)$ & 0.01 \\
\hline \multicolumn{6}{|l|}{$n-6$} \\
\hline d5-20:3 & $1.62(0.66-2.55)$ & $2.39(1.26-3.83)$ & 0.97 & $1.36(0.46-3.82)$ & 0.23 \\
\hline d5-20:4 & $0.41(0.15-0.95)$ & $0.80(0.40-1.38)$ & 0.11 & $0.46(0.27-1.57)$ & 0.31 \\
\hline
\end{tabular}

$\S$ Data presented as median (p25-p75) of the integrated area under the plasma concentration-time course curve (AUC) over the 96 hours of the study (arbitrary units).

* $p$ values obtained using non parametric test (Kruskal-Wallis).

the two control groups did not reach statistical significance. However, for DHA, the value is significantly lower in the IUGR group; it is approximately one third that observed in the birth weight-matched control infants.

The ratios of products to precursors may be used to compare differences in metabolism across groups. The AUC values presented in Table 3 were used to compute these ratios. This parameter provides some insight into the relative formation of AA and DHA in the IUGR and the two reference groups. The result of product:precursor ratios using the AUC values for the d5-labeled n- 6 fatty acids does not reveal differences between IUGR and either reference group (data not shown). The AUC values for the various isotopic fatty acids of the $n-6$ series were similar across all groups.

The results of product:precursor ratios for the n-3 pathway that form DHA are shown in Fig. 1. There are significantly lower values in the IUGR group relative to the control groups for DHA formed from all three main precursors (Fig. 1A). For DHA formation from LNA, the difference is significant for the birth weight-matched control group but not for the GA control group $(p=0.07)$. The ratio of DHA to LNA is 2-fold greater in

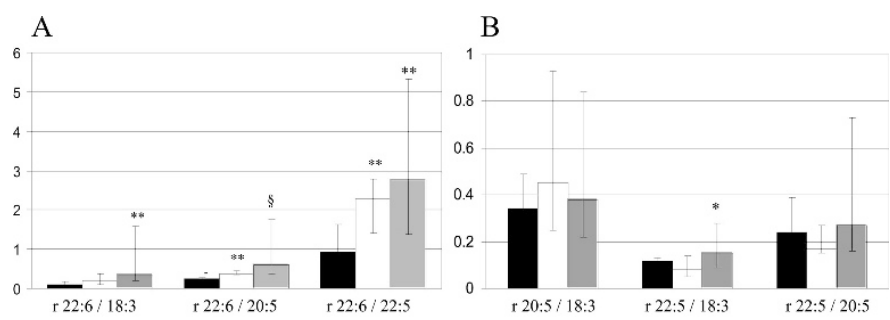

Figure 1. Product:precursor ratio after integration of the d5-labeled n-3 fatty acid plasma concentration-time course curves. This figure depicts the product:precursor ratios using the AUCs of the respective plasma concentration-time course curves. (A) From left to right, the ratios of the product of interest d5-DHA (d5-22:6 n-3) to the precursor d5-LNA (d5 18:3 n-3) and the intermediate metabolites d5-EPA (d5-20:5 n-3) and d5-DPA (d5-22:5 n-3). (B) From left to right, the ratios along the $n-3$ series metabolic pathway of d5-EPA (d5-20:5 n-3) and d5-DPA (d5-22:5 n-3) from the parental EFA d5-LNA (d5-18:3 n-3) and the d5-EPA (d5-20:5 n-3) to d5-DPA (d5-22:5 n-3) ratio. Bars represent the median and interquartile (p25-p75) range. Symbols over the bars indicate a significant difference when comparing $\operatorname{IUGR}(\mathbf{\square}, \mathrm{n}=11)$ with GA- $(\square, \mathrm{n}=13)$ and birth weight $(\square, \mathrm{n}=25)$-matched control groups using Kruskal-Wallis. $* p<0.05 ; * * p<0.01 ; \S p<0.001$. the GA control infants and 3-fold higher in the birth weight control infants relative to the infants with IUGR. We performed analysis of covariance to assess the effect of differences in gender between the IUGR and birth weight-matched control groups in the reported finding. The $p$ value for the observed effect of IUGR in the evaluated isotopic DHA:LNA, DHA:EPA, and DHA:DPA ratios remain significant after adding gender to each DHA/ precursor model.

Figure $1 B$ examines the portion of the metabolic pathway that leads from LNA to EPA and DPA. There were no differences in the ratios of EPA:LNA or of DPA:LNA for the IUGR versus the GA matched control infants. However, there was an increased ratio for the DPA:LNA for the birth weight group relative to the IUGR group. The elongation step forming DPA from EPA seems to be relatively insensitive to birth weight or GA. The large differences in the DHA:DPA ratio indicate that this may be the metabolic step that is most restricted in infants with IUGR.

\section{DISCUSSION}

The present work addresses specifically LCP metabolism in infants with IUGR compared with neonates of equivalent birth weight but shorter gestation or similar gestation but who are significantly smaller at birth. Our current results suggest lower formation of DHA in infants with IUGR and complement our previous observations $(10,15)$ and those of others $(9,20)$ that demonstrate higher LCP formation in neonates of lower birth weight and AGA. From these studies, we have been able to show that term and preterm infants synthesize DHA and AA from LNA and LA and that preterm infants seem to have an overall metabolic conversion greater than that found in term infants. The decrease in DHA and not in AA formation in infants with IUGR suggests that the metabolic abnormality may be related to the final metabolic steps that include the insertion of the sixth double bond and a partial $\beta$-oxidation to form DHA, because AA formation does not require these steps in its metabolism. The relative conversions observed in the n-3 pathway as shown in Fig. 1A support this concept, because it seems that the most restricted step is conversion of DPA to DHA. This conversion includes an elongation, a $\Delta 6$ desaturation, and a partial $\beta$-oxidation, which is considered to occur 
mainly in the peroxisome (21). On the basis of this finding, we speculate that infants with IUGR may have an impaired peroxisomal $\beta$-oxidation that explains the lower DPA to DHA conversion observed in this group of infants compared with the AGA control groups.

There is evidence that neonates with IUGR have altered maturation or inactive enzymes related to lipid metabolism $(2,3,22)$. The work of Lane et al. (22) using rat models with uteroplacental insufficiency documents that the expression of the peroxisome proliferator-activated receptor (PPAR)- $\gamma$ coactivator-1 (PGC-1) mRNA varies according to muscle groups, suggesting differences in oxidative capacity of muscle fibers. The PGC-1 expression is gender specific. Higher expression of carnitine palmitoyl transferase I, an enzyme that regulates fatty acid $\beta$-oxidation as well as its transcriptional co-activator PGC-1, has also been documented in the skeletal muscle of rats with IUGR (22). These results highlight evidence of impaired expression of key enzymes in lipid metabolism that are mediated by the family of the nuclear receptor PPARs (PPAR- $\gamma$, PPAR- $\alpha$, and PPAR- $\beta$ ). This group of transcriptional factors is known by its important regulatory effect on adiposity differentiation and lipid metabolism $(23,24)$ and offers a possible explanation to the limited peroxisomal $\beta$-oxidation suggested by our results.

Considering the important role that DHA plays in neural and retinal development, the finding of a lower formation of DHA in infants with IUGR may have important implications as it can be a contributing factor to the abnormalities in neurodevelopment described in infants with IUGR $(25,26)$. Also, DHA supplementation may improve DHA status in infants with a demonstrated DHA deficiency, such as premature infants or those with Zellweger syndrome. Taken together, these findings suggest a potential benefit for early nutritional interventions with DHA supplementation to improve neurologic outcome for infants with IUGR. Several clinical studies have shown the benefits of DHA supplementation in visual function of premature infants, improvement that correlates with better DHA status in red blood cells and plasma $(27,28)$ More recently, Sarkadi-Nagy et al. (29) were able to document a positive correlation between DHA supplementation and DHA content in the brain, retina, and liver in 4-wk-old (adjusted age) baboons. The work of Martinez et al. (30) on 13 patients who had Zellweger and whose vision, muscle tone, social contact, and liver function and brain myelination improved after being supplemented with DHA ethyl ester (100-500 mg/d), offers further evidence in favor of the effect of DHA supplementation in patients with a DHA deficit.

There is an important difference in the feeding status of the infants with IUGR and the two control groups at the time of study. The effect of this difference in the reported results is difficult to assess because of limitations imposed by the small sample size. Nevertheless, that none of the patients in the IUGR group had received DHA-containing milk formula makes it unlikely that differences in the type of feeding explain the finding of lower DHA formation in infants with IUGR compared with the two AGA control groups. The possible effect of the age difference at study entrance between the infants with IUGR and the birth weight-matched control group on the results works in the opposite direction of the observed effects, because it would favor a higher DHA formation in the infants with IUGR compared with the $1 \mathrm{wk}$ postnatally older birth weight-matched infants $(10,20)$.

Finally, we consider selected methodologic aspects to be taken into account in the analysis of results. The simple model of AUC integration assumes that we are able to model concentrations over time using a linear interpolation between data points forming a trapezoid. Thus, we will underestimate the area when data points form a convex curve and conversely will overestimate the area when the curve is concave. The limited number of samples obtained in this study during the initial $24 \mathrm{~h}$ will predictably underestimate the precursor concentrations over time. There was also an underestimation of product formation because the study was restricted to $96 \mathrm{~h}$. In our more recent studies, we have verified that, in some cases, DHA appearance in the plasma peaks at $\sim 96 \mathrm{~h}$ but may continue for up to $168 \mathrm{~h}$. This could account in part for the apparent lower rate of DHA formation observed in infants with IUGR; it is clear that there is a slower decline in LNA and a slower rate of rise in DHA on the basis of the observed isotopic fatty acid concentrations at 24,48 , and $96 \mathrm{~h}$. Thus, perhaps the process may be slower than normal for IUGR but cumulative DHA formation over time may be unchanged. The limited blood sampling possible in this study does not allow a more complex kinetic analysis of fatty acids considering the fractional rate of synthesis and catabolism. The in vivo physiology and biochemistry of LCP metabolism are sufficiently complex such that even with presently available stable isotope methods, care must be exercised in the interpretations of the parameters available. The combined use of radioactive isotopes $\left(\left[1-{ }^{11} \mathrm{C}\right] \mathrm{AA}\right.$ and $\left.\left[1-{ }^{11} \mathrm{C}\right] \mathrm{DHA}\right)$ and in vivo detection of labeled metabolites using positron emission topography may permit in the future a more dynamic evaluation of DHA metabolism coupled to specific anatomic/functional loci within the CNS and specific cognitive functions (31). That stable isotope studies are based on the plasma pool has been considered an important limitation of this type of studies on the basis of the idea that plasma fatty acid status may not necessarily reflect the LCP accretion of critical organs such as the CNS and the retina $(29,32,33)$.

\section{CONCLUSION}

In conclusion, this study extends our previous report of enhanced DHA and AA formation in neonates with lower GA and birth weight indicating that less mature infants with adequate fetal growth have greater LCP formation from EFA precursors. The finding of significantly lower DHA formation in the infants with IUGR relative to neonates who were $1 \mathrm{~kg}$ greater in birth weight is in contrast to the prediction based on the observed enhanced metabolic activity in preterm infants of lower birth weight. That this restriction occurs only in the formation of DHA and not of AA suggests that the effect may be related to metabolic steps that are specific to DHA. Our results lead us to suggest that infants with IUGR have a specific impairment in the final biosynthetic pathway that leads to the synthesis of DHA. We speculate that this metabolic defect 
could contribute to the abnormalities in neurodevelopment observed in neonates with IUGR.

\section{REFERENCES}

1. Pardi G, Cetin I, Marconi AM, Lanfranchi A, Bozzetti P, Ferrazzi E, Buscaglia M, Battaglia FC 1993 Diagnostic value of blood sampling in fetuses with growth retardation. N Engl J Med 328:692-696

2. Lane RH, MacLennan NK, Hsu JL, Janke SM, Pham TD 2002 Increased hepatic peroxisome proliferator-activated receptor-gamma coactivator-1 gene expression in a rat model of intrauterine growth retardation and subsequent insulin resistance. Endocrinology 143:2486-2490

3. Lane RH, MacLennan NK, Daood MJ, Hsu JL, Janke SM, Pham TD, Puri AR, Watchko JF 2003 IUGR alters postnatal rat skeletal muscle peroxisome proliferatoractivated receptor-gamma coactivator-1 gene expression in a fiber specific manner. Pediatr Res 53:994-1000

4. Cetin I, Giovannini N, Alvino G, Agostoni C, Riva E, Giovannini M, Pardi G 2002 Intrauterine growth restriction is associated with changes in polyunsaturated fatty acid fetal-maternal relationships. Pediatr Res 52:750-755

5. Felton CV, Chang TC, Crook D, Marsh M, Robson SC, Spencer JA 1994 Umbilical vessel wall fatty acids after normal and retarded fetal growth. Arch Dis Child Feta Neonatal Ed 70:F36-F39

6. Percy P, Vilbergsson G, Percy A, Mansson JE, Wennergren M, Svennerholm L 1991 The fatty acid composition of placenta in intrauterine growth retardation. Biochim Biophys Acta 1084:173-177

7. Vilbergsson G, Samsioe G, Wennergren M, Karlsson K 1991 Essential fatty acids in pregnancies complicated by intrauterine growth retardation. Int J Gynaecol Obste 36:277-286

8. Rump P, Mensink RP, Kester AD, Hornstra G 2001 Essential fatty acid composition of plasma phospholipids and birth weight: a study in term neonates. Am J Clin Nutr 73:797-806

9. Sauerwald TU, Hachey DL, Jensen CL, Chen H, Anderson RE, Heird WC 1997 Intermediates in endogenous synthesis of C22:6 omega 3 and C20:4 omega 6 by term and preterm infants. Pediatr Res 41:183-187

10. Uauy R, Mena P, Wegher B, Nieto S, Salem N Jr 2000 Long chain polyunsaturated fatty acid formation in neonates: effect of gestational age and intrauterine growth. Pediatr Res 47:127-135

11. Ballard JL, Khoury JC, Wedig K, Wang L, Eilers-Walsman BL, Lipp R 1991 New Ballard Score, expanded to include extremely premature infants. J Pediatr 119:417423

12. Lubchenco LO, Hansman C, Dressler M, Boyd E 1963 Intrauterine growth as estimated from liveborn birth-weight data at 24 to 42 weeks of gestation. Pediatrics 32:793-800

13. Metcoff J 1994 Clinical assessment of nutritional status at birth. Fetal malnutrition and SGA are not synonymous. Pediatr Clin North Am 41:875-891

14. Milad M, Mena P, Nieto S, Uauy R 2004 Fatty acid composition of human milk lipids in Chilean women. Acta Paediatr 93:855-856
15. Salem N Jr, Wegher B, Mena P, Uauy R 1996 Arachidonic and docosahexaenoic acids are biosynthesized from their 18-carbon precursors in human infants. Proc Nat Acad Sci USA 93:49-54

16. Folch J, Lees M, Sloane Stanley GH 1957 A simple method for the isolation and purification of total lipides from animal tissues. J Biol Chem 226:497-509

17. Morrison WR, Smith LM 1964 Preparation of fatty acid methyl esters and dimethylacetals from lipids with boron fluoride-methanol. J Lipid Res 53:600-608

18. Salem N Jr, Reyzer M, Karanian J1996 Losses of arachidonic acid in rat liver after alcohol inhalation. Lipids 31:S153-S156

19. Yeh KC, Kwan KC 1978 A comparison of numerical integrating algorithms by trapezoidal, Lagrange, and spline approximation. J Pharmacokinet Biopharm 6:79-98

20. Sauerwald TU, Hachey DL, Jensen CL, Heird WC 1997 New insights into the metabolism of long chain polyunsaturated fatty acids during infancy. Eur J Med Res 2:88-92

21. Voss A, Reinhart M, Sankarappa S, Sprecher H 1991 The metabolism of 7,10,13,16,19-docosapentaenoic acid to 4,7,10,13,16,19-docosahexaenoic acid in rat liver is independent of a 4-desaturase. J Biol Chem 266:19995-20000

22. Lane RH, Kelley DE, Ritov VH, Tsirka AE, Gruetzmacher EM 2001 Altered expression and function of mitochondrial beta-oxidation enzymes in juvenile intrauterine-growth-retarded rat skeletal muscle. Pediatr Res 50:83-90

23. Uauy R, Mena P, Rojas C 2000 Essential fatty acids in early life: structural and functional role. Proc Nutr Soc 59:3-15

24. Jump DB 2002 Dietary polyunsaturated fatty acids and regulation of gene transcription. Curr Opin Lipidol 13:155-164

25. Brandt I, Sticker EJ, Lentze MJ 2003 Catch-up growth of head circumference of very low birth weight, small for gestational age preterm infants and mental development to adulthood. J Pediatr 142:463-468

26. Hack M, Breslau N, Weissman B, Aram D, Klein N, Borawski E 1991 Effect of very low birth weight and subnormal head size on cognitive abilities at school age. N Engl J Med 325:231-237

27. Birch EE, Birch DG, Hoffman DR, Uauy R 1992 Dietary essential fatty acid supply and visual acuity development. Invest Ophthalmol Vis Sci 33:3242-3253

28. Faldella G, Govoni M, Alessandroni R, Marchiani E, Salvioli GP, Biagi PL, Spano C 1996 Visual evoked potentials and dietary long chain polyunsaturated fatty acids in preterm infants. Arch Dis Child Fetal Neonatal Ed 75:F108 -F112

29. Sarkadi-Nagy E, Wijendran V, Diau GY, Chao AC, Hsieh AT, Turpeinen A, Nathanielsz PW, Brenna JT 2003 The influence of prematurity and long chain polyunsaturate supplementation in 4-week adjusted age baboon neonate brain and related tissues. Pediatr Res 54:244-252

30. Martinez M, Vazquez E, Garcia-Silva MT, Manzanares J, Bertran JM, Castello F, Mougan I 2000 Therapeutic effects of docosahexaenoic acid ethyl ester in patients with generalized peroxisomal disorders. Am J Clin Nutr 71:376S-385S

31. Rapoport SI 2003 In vivo approaches to quantifying and imaging brain arachidonic and docosahexaenoic acid metabolism. J Pediatr 143:S26-S34

32. Blank C, Neumann MA, Makrides M, Gibson RA 2002 Optimizing DHA levels in piglets by lowering the linoleic acid to alpha-linolenic acid ratio. J Lipid Res 43:1537-1543

33. Carver JD, Benford VJ, Han B, Cantor AB 2001 The relationship between age and the fatty acid composition of cerebral cortex and erythrocytes in human subjects. Brain Res Bull 56:79-85 